\title{
Placental Endothelial Nitric Oxide Synthase in Multiple and Single Dose Betamethasone Exposed Pregnancies
}

\author{
Heather L. MERTZ, M.D., Lisa MELE, Sc. M., Catherine Y. SPONG, M.D., Donald J. DUDLEY, \\ M.D., Ronald J. WAPNER, M.D., Jay D. IAMS, M.D., Yoram SOROKIN, M.D., Alan \\ PEACEMAN, M.D., Kenneth J. LEVENO, M.D., Steve N. CARITIS, M.D., Menachem \\ MIODOVNIK, M.D., Brian M. MERCER, M.D., John M. THORP, M.D., Mary J. O'SULLIVAN, \\ M.D., Susan M. RAMIN, M.D., Marshall CARPENTER, M.D., Dwight J. ROUSE, M.D., and \\ Baha SIBAI, M.D. for the Eunice Kennedy Shriver National Institute of Child Health and \\ Human Development Maternal-Fetal Medicine Units Network \\ Departments of Obstetrics and Gynecology from the Wake Forest University Health Sciences, \\ Winston-Salem, NC; University of Utah, Salt Lake City, UT; Drexel University, Philadelphia, PA; \\ The Ohio State University, Columbus, OH; Wayne State University, Detroit, MI; Northwestern \\ University, Chicago, IL; University of Texas Southwestern Medical Center, Dallas, TX; University \\ of Pittsburgh, Pittsburgh, PA; Columbia University, New York, NY; University of Cincinnati, \\ Cincinnati, $\mathrm{OH}$; Case Western Reserve University, Cleveland, $\mathrm{OH}$; University of North Carolina, \\ Chapel Hill, NC; University of Miami, Miami, FL; The University of Texas Health Science Center \\ at Houston, Houston, TX; Brown University, Providence, RI; University of Alabama at \\ Birmingham, Birmingham, AL; University of Tennessee, Memphis, TN; and The George \\ Washington University Biostatistics Center, Washington, DC and the Eunice Kennedy Shriver \\ National Institute of Child Health and Human Development, Bethesda, MD
}

\section{Abstract}

Objective-To compare endothelial nitric oxide synthase (eNOS ) expression and capillary density (CDS) in placentas exposed to single or multiple courses of betamethasone.

Study design-Placental specimens exposed to single versus repeat courses of betamethasone were analyzed through immunohistochemistry and digital image quantification for eNOS and CD34. Quantified eNOS staining, calculated CDS, ratio of eNOS to CDS, and clinical characteristics were compared. Linear regression performed with these as dependent variables.

\begin{abstract}
Results-Mean and maximum CDS were increased ( $\mathrm{p}=0.013$ and 0.005$)$ and the ratio of eNOS to CDS decreased $(\mathrm{p}=0.016)$ in specimens exposed to 4 courses of betamethasone compared with 1 to 3 courses. Exposure to 4 courses of betamethasone was associated with increased CDS, but not with eNOS expression.
\end{abstract}

Conclusion-Exposure to 4 courses of betamethasone is associated with increased placental CDS. The placental effects of multiple courses of betamethasone are unrelated to eNOS expression.

(C) 2011 Mosby, Inc. All rights reserved.

Correspondence: Heather L. Mertz, M.D., Assistant Professor, Section on Maternal-Fetal Medicine, Department of Obstetrics and Gynecology, Wake Forest University, Medical Center Boulevard, Winston-Salem, NC 27157, hmertz@ wfubmc.edu, Telephone: (336) 716-1025, Fax: (336) 716-6937.

Publisher's Disclaimer: This is a PDF file of an unedited manuscript that has been accepted for publication. As a service to our customers we are providing this early version of the manuscript. The manuscript will undergo copyediting, typesetting, and review of the resulting proof before it is published in its final citable form. Please note that during the production process errors may be discovered which could affect the content, and all legal disclaimers that apply to the journal pertain. 


\section{Keywords}

betamethasone; eNOS; placenta

\section{INTRODUCTION}

Multiple courses of antenatal betamethasone have been shown to result in decreased birthweight and neonatal head circumference. ${ }^{1,2}$ However, the mechanism of this effect remains unclear.

In studies examining fetal growth restriction, it has been found that the large increase in fetal growth during the last half of pregnancy depends primarily on a corresponding increase in transplacental exchange. ${ }^{3,4}$ This occurs through coordination of angiogenesis, adaptations of vascular tone, and efficiency of exchange and is supported by significant growth of the placental vascular beds with resultant increases in uterine and umbilical blood flow. ${ }^{5,6}$ It has further been shown that the vascular density of fetal placental components increases dramatically during the last third of gestation as compared with maternal components which have been shown to have a consistent slow increase throughout gestation. $6,7,8$

Endothelial nitric oxide synthase (eNOS) is an important component of regulation of vascular tone and blood flow and has been linked to feto-placental growth and development. Several authors have postulated that this is a result of eNOS mediated nitric oxide release from the fetal tissues and is supported by immunoreactive and in situ hybridization demonstration of eNOS expression by extravillous trophoblast cells. ${ }^{9,10}$

Additionally, antenatal glucocorticoid exposure in primates has been shown to significantly reduce eNOS protein as well as other indices of eNOS function. This effect has been seen in both the syncytiotrophoblast as well as the vascular endothelium. ${ }^{9,11}$

The objective of this study was to compare eNOS expression and capillary density in placentas from pregnancies exposed to multiple or single courses of betamethasone. It was our hypothesis that betamethasone exposure influences fetal growth through a nitric oxide mediated mechanism.

\section{MATERIALS AND METHODS}

\section{Study design}

This study is an analysis of placental specimens collected during the conduct of a randomized multicenter trial that compared the effect of administering single versus weekly repeat courses of antenatal betamethasone in preventing neonatal morbidity. The trial was conducted between March 2000 and April 2003 at participating centers of the the Eunice Kennedy Shriver National Institute of Child Health and Human Development, MaternalFetal Medicine Units ( MFMU) Network. The primary study has been previously described. ${ }^{1}$ Participants in the trial were pregnant women between 23 weeks 0 days and 31 weeks 6 days, who were at risk for spontaneous preterm delivery. Women with preterm premature rupture of the membranes (PPROM) prior to randomization, confirmed fetal lung maturity, chorioamnionitis, a major fetal anomaly, non-reassuring fetal status, systemic corticosteroid use during the current pregnancy, or insulin-dependent diabetes were excluded.

Patients were randomized to receive weekly courses of betamethasone or placebo, one week after receiving a single full course of corticosteroids (betamethasone or dexamethasone). 
Each course consisted of two injections of betamethasone $12 \mathrm{mg}$ (as $6 \mathrm{mg}$ betamethasone sodium phosphate and $6 \mathrm{mg}$ betamethasone acetate) repeated once in 24 hours or matching placebo. Initially, patients received courses until delivery or 33 weeks 6 days' gestation, whichever was sooner. After 67 patients had been enrolled, the number of courses (not including the qualifying course) was limited to 4 because of difficulty in recruitment and because of accumulating literature suggesting possible harmful effects of multiple courses.

Placentas were collected at the time of delivery. For this analysis, placental specimens from patients with singleton pregnancies were evaluated.

\section{Placental specimen preparation}

Specific samples were obtained from each placental specimen and were formalin fixed and paraffin embedded. All samples included in this analysis were from a standardized lateral location within the placenta containing both maternal and fetal surfaces. Institutional Review Board approval was obtained before conducting this investigation.

After obtaining the paraffin blocks of these placental tissues, sections were cut at four microns, adhered to ProbeOn Plus slides from a protein-free water bath and air-dried overnight. Slides were then deparaffinized and hydrated to deionized water. Following HIER (Heat Induced Epitope Retrieval) in Citrate buffer pH 6.0 for 30 minutes using a Black and Decker rice steamer (eNOS/NOS type III, BD Transduction Laboratories) and enzymatic retrieval using Trypsin at $1 \mathrm{mg} / \mathrm{ml}, 4 \mathrm{mM} \mathrm{CaCl}_{2}$ in $20 \mathrm{mM}$ TRIS $\mathrm{pH} 7.7$ for 30 minutes at $37^{\circ} \mathrm{C}$, slides were washed for five minutes under running deionized water, then transferred to a hand-held MicroProbe slide holder. A $1 X$ Automation Buffer (Biomeda) containing $0.1 \%$ Tween 20 to eliminate surface tension and $0.5 \%$ Casein (Sigma) to blocking non-specific background, was used both as wash buffer and antibody diluent. Serial sections were incubated with mouse monoclonal eNOS/NOS Type III (BioGenex) at 1:50 and monoclonal CD34 (Novocastra) at 1:10 overnight at $4{ }^{\circ} \mathrm{C}$. Following several washes with $1 \mathrm{X}$ buffer, slides were incubated with biotinylated anti-mouse antibodies (BioGenex) at 1:20 for 20 minutes at $37^{\circ} \mathrm{C}$. Following additional washes using $1 \mathrm{X}$ buffer, sections were incubated with a Streptavidin-alkaline phosphatase complex (BioGenex) at 1:20 for 20 minutes at $37^{\circ} \mathrm{C}$. The red end product was visualized using a Vector Red chromogen (Vector Laboratories). Slides were counterstained for five minutes in Mayer's hematoxylin, washed under running deionized water, dehydrated using graded alcohols, cleared through p-xylene and mounted with Permount (Figure 1, Figure 2). This technique is applied routinely to paraffin embedded specimens in both clinical and research immunohistochemistry laboratories and the antibodies employed in this investigation were specifically noted for use on paraffin sections. ${ }^{12}$ The HIER process described above enhances this technique and consists of enzymatic retrieval of protein cross linkages that might have occurred through formalin fixation in these tissues, allowing return to a native configuration of any proteins that may have been altered during fixation. ${ }^{13}$

Digital images of each slide were then created and staining was quantified for both eNOS and CD34 using Image Pro Plus software. This quantification was based on the number of pixels in each digital image exhibiting immunostaining (Figure 3). Each micrometer stained represented 2.160 pixels in this model. The slides were analyzed in quadrants with measurements taken from all four quadrants for each specimen. A mean was also calculated for each specimen from these four measurements.

Capillary area density, defined as the CD34 staining area as a proportion of total field area, was then calculated for each specimen. Total field area for the slides employed in this study was found to be 76,3202.1 micrometers. This is a well accepted approach that has been used to quantitate angiogenesis in placental tissues. This has also been correlated with expression 
of angiogenic factors. ${ }^{14,15,16}$ An additional parameter, the ratio of eNOS to capillary area density was calculated for each specimen utilizing mean eNOS staining and mean capillary area density values. All investigators remained blinded to the clinical group assignment of the specimens until all immunohistochemistry and staining quantification had been completed.

\section{Statistical analysis}

The main outcomes for this analysis were capillary area density, eNOS expression, and the ratio of eNOS to capillary area density. Treatment and placebo groups were compared. Patients in each study arm who received one to three study courses versus four study courses were also examined. Chi-square or Fisher's Exact test was used to compare categorical variables and the Wilcoxon Rank Sum test was used to compare continuous variables. Linear regression models in which the effect of treatment group exposure to four study courses and gestational age at delivery with the outcomes of capillary area density and eNOS were performed.

Additional regression analysis of patients assigned to the active group which examined the effect of exposure to 4 study courses versus one to three courses was also performed. Possible confounding variables included in these models were maternal age, parity, ethnicity, maternal body mass index, maternal level of education, gestational age at randomization, gestational age at delivery, tobacco use, presence of preterm labor, exposure to tocolytics, maternal infection, preterm premature rupture of membranes, and presence of placental calcification and placental infarction recorded as present or absent in the initial pathology exams of these placentas.

A nominal $\mathrm{P}$ value of less than 0.05 was considered to indicate statistical significance, and no adjustments were made for multiple comparisons.

\section{RESULTS}

There were 191 placental specimens able to be completely analyzed. The active treatment group, receiving multiple courses of betamethasone, represented 100 and the placebo group, receiving a single course of betamethasone, represented 91 of these samples. Of those in the active treatment group, 68 had exposure to 4 courses of antenatal betamethasone, 32 had exposure to 1 to 3 study courses. Baseline clinical characteristics for patients receiving single and multiple courses of betamethasone were similar (Table 1).

There were no significant differences in the mean or maximum capillary area density or in the mean and maximum quantified eNOS expression between the active and the placebo groups (Table 2). When results were stratified by number of study courses, mean and maximum capillary area density were increased (Table 3 ) and the ratio of eNOS to capillary area density was decreased (Table 3), in patients in the active group exposed to 4 study courses compared with those who received 1 to 3 courses. Mean and maximum eNOS staining was not significantly different between these groups.

The association of treatment group, exposure to four study courses, and gestational age at delivery with the outcomes eNOS, capillary density, and ratio of eNOS to capillary density was examined using linear regression analysis. There was a significant association between the interaction of treatment group and exposure to four study courses and capillary density $(\mathrm{p}=0.047)$. Gestational age at delivery was directly associated with capillary density $(\mathrm{p}=0.0025)$ and inversely associated with eNOS expression $(\mathrm{p}=0.031)$ and the ratio of eNOS expression to capillary density $(\mathrm{p}=0.0009)$. 
Regression analysis was performed for those who received four study courses, either active or placebo which adjusted for possible confounding factors including maternal age, parity, ethnicity, maternal body mass index, maternal level of education, gestational age at randomization, gestational age at delivery, tobacco use, presence of preterm labor, exposure to tocolytics, maternal infection, preterm premature rupture of membranes, and presence of placental calcification and placental infarction. This analysis showed that exposure to four courses of betamethasone was significantly associated with increased capillary density $(\mathrm{p}=0.018)$ compared with receipt of a single course. Capillary density was also significantly associated with gestational age at randomization $(\mathrm{p}=0.004)$ and maternal age $(\mathrm{p}=0.015)$.

Further regression analysis of the active treatment group which adjusted for the same possible confounding factors listed above showed that exposure to four courses of betamethasone was significantly associated with increased capillary density $(\mathrm{p}=0.0075)$ compared with 1 to 3 courses. Increasing maternal age was also associated with increased capillary density $(\mathrm{p}=0.021)$.

\section{COMMENT}

This investigation examines human placental eNOS in the setting of a clinical trial comparing single to multiple courses of betamethasone. The rigorous study design of the clinical trial preceding this investigation and the volume of samples included are clear strengths of this analysis.

Our findings likely indicate that the feto-placental effects of antenatal betamethasone exposure occurs independent of the influences of eNOS expression. We did not find differences in mean and maximum eNOS staining in those placentas exposed to single as compared with multiple antenatal courses of betamethasone. This finding persisted even when results were stratified according to specific number of betamethasone courses.

In pregnancies exposed to 4 antenatal betamethasone courses, mean and maximum capillary density was increased as compared to those exposed to 1 to 3 courses. Further, the ratio of eNOS to capillary density decreased in these specimens, confirming the unlikely influence of eNOS in these processes. This suggests that there may be other regulators of angiogenesis that are affected by antenatal betamethasone exposure to four courses of antenatal betamethasone were the ones found to have the most pronounced effects on fetal growth (1). These data support a nitric oxide independent compensatory mechanism to stimulate capillary growth when the feto-placental unit is exposed to multiple courses of antenatal betamethasone or other similar insults.

However, despite increased capillary density, pregnancies exposed to 4 courses of antenatal betamethasone were the ones found to have the most pronounced effects on fetal growth. ${ }^{1}$ This suggests an angiogenic response to repeated betamethasone exposure that is not able to improve exchange within the feto-placental unit and questions the functional integrity of these vessels.

We also found that increasing gestational age was associated with decreasing placental eNOS expression and increasing capillary density. These findings have been suggested in other similar investigations in mammalian species. ${ }^{17,18}$ Our study has uniquely focused on examining eNOS as it is related to capillary density in human placentas.

Additionally, we found that increasing maternal age was associated with increased placental capillary density. The clinical significance of this is uncertain at the present time but again suggests that there may be other important compensatory mechanisms in place that influence placental angiogenesis in the particular circumstance of increasing maternal age and 
exposure to multiple antenatal courses of betamethasone. The question also arises as to whether the effects of maternal age on the feto-placental unit may be similar to those of exposure to multiple antenatal courses of betamethasone. This is supported by recent reports of an association between advancing maternal age and poor pregnancy outcomes, including fetal growth restriction. ${ }^{19,20}$

This study has several limitations. The most significant of which is that we do not have data from these specimens that includes Western blotting. Thus, we are unable to make comparisons between our immunohistochemical staining and the results of Western blotting in these specimens. Additionally, regional differences may exist within the placenta. We have studied a single lateral placental location in these specimens and while this was standardized for every specimen, it could be postulated that differences in other locations within the placental bed may exist.

The regulation and influence of eNOS is complex and immunohisotochemistry at a single clinical point in time may not be able to adequately account for variations through gestation. It is likely that placental eNOS may have influences that precede the findings at delivery and that are more pronounced earlier in gestation. Our results support this as eNOS expression in these specimens was inversely related to gestational age. The results of other investigators confirm this relationship as well as the complex interactions of eNOS within the maternal and fetal compartments. ${ }^{21,22}$

In summary, this investigation provides novel information regarding the placental effects of antenatal exposure to multiple as compared with single courses of betamethasone. While eNOS does not appear to be involved in these effects, there does seem to be an important influence on placental angiogenesis in the setting of exposure to four courses of antenatal betamethasone. Ongoing investigation is needed to more clearly elucidate the mechanisms of the effects of antenatal betamethasone exposure on the feto-placental unit. Such investigations have the potential to give further insight into the mechanisms of the effects of betamethasone exposure during pregnancy as well as normal and abnormal fetal growth.

\section{Acknowledgments}

The author thanks the following Network members who participated in protocol development and coordination between clinical research centers (Michelle DiVito, M.S.N. and Francee Johnson, R.N., B.S.N), protocol/data management and statistical analysis (Elizabeth Thom, Ph.D.), and protocol oversight (Margaret Harper, M.D., M.Sc.).

Supported by grants from the Eunice Kennedy Shriver National Institute of Child Health and Human Development (HD21410, HD21414, HD27869, HD27917, HD27905, HD27860, HD27861, HD27915, HD34122, HD34116, HD34208, HD34136, HD40500, HD40485, HD40544, HD40545, HD40560, HD40512, HD36801) and M01RR-000080 from the National Center for Research Resources (NCRR) and its content is solely the responsibility of the authors and does not necessarily represent the official views of the NICHD, the National Institutes of Health, and the NCRR.

\section{References}

1. Wapner RJ, Sorokin Y, Thom EA, et al. Single versus weekly courses of antenatal corticosteroids: Evaluation of safety and efficacy. Am J Obstet Gynecol. 2006; 195:633-642. [PubMed: 16846587]

2. French NP, Hagan R, Evans SF, et al. Repeated antenatal corticosteroids: Size at birth and subsequent development. Am J Obstet Gynecol. 1999; 180:114-121. [PubMed: 9914589]

3. Arroyo JA, Winn VD. Vasculogenesis and angiogenesis in the IUGR placenta. Semin Perinatol. 2008; 32(3):172-177. [PubMed: 18482617]

4. Torry DS, Hinrichs M, Torry RJ. Determinants of placental vascularity. Am J Reprod Immunol. 2004; 51:257-268. [PubMed: 15212678] 
5. Burton GJ, Charnock-Jones DS, Jauniaux E. Regulation of vascular growth and fundtion in the human placenta. Reproduction. 2009; 138:895-902. [PubMed: 19470597]

6. Mayhew TM. Fetoplacental angiogenesis during gestation is biphasic, longitudinal and occurs by proliferation and remodeling of vascular endothelial cells. Placenta. 2002; 23:127-139.

7. Reynolds LP, Redmer DA. Angiogenesis in the placenta. Biol Reprod. 2001; 64:1033-1040. [PubMed: 11259247]

8. Reynolds LP, Redmer DA. Utero-placental vascular development and placental function. J Anim Sci. 1995; 73:1839-1851. [PubMed: 7545661]

9. Myatt L, Brewer A, Brockman DE. The action of nitric oxide in the perfused human fetal-placental circulation. Am J Physiol. 1991; 164:687-692.

10. Martin D, Conrad KP. Expression of endothelial nitric oxide synthase by extravillous trophoblast cells in the human placenta. Placenta. 2000; 21:23-31. [PubMed: 10692247]

11. Aida K, Wang XL, Wang J, et al. Effect of betamethasone administration to the pregnant baboon at 0.75 gestation on placental eNOS distribution and activity. Placenta. 2004; 25:780-787. [PubMed: 15451192]

12. Van der Loos, CM. Immunoenzyme Multiple Staining Methods. Springer-Verlag New York Inc; New York, NY: 1999.

13. Shi, S. Antigen Retrieval Techniques: Immunohistochemisty and Molecular Morphology. Eaton Publishing, BioTechniques Books Division; Natick, MA: 2000.

14. Reynolds LP, Borowicz PP, Vonnahme KA, et al. Placental angiogenesis in sheep models of compromised pregnancy. J Physiol. 2005; 565.1:43-58. [PubMed: 15760944]

15. Burton GJ, Rashnetnikova OS, Milovanov AP, et al. Stereologic evaluation of vascular adaptations in human placental villi to differing forms of hypoxic stress. Placenta. 1996; 17:49-55. [PubMed: 8710813]

16. Borowicz PP, Johnson ML, Grazul-Bilska AT, et al. Modeling the relationship among quantitative measures of placental angiogenesis and expression of mRNA for vascular endothelial growth factor, angiopoietins, and their receptors. Biol Reprod. 2004; 70(Suppl 1):207.

17. Vatish M, Randeva HS, Grammatopoulos DK. Hormaonal regulation of placental nitric oxide and pathogenesis of preeclampsia. Trends in Molecular Medicine. 2006; 12:223-233. [PubMed: 16616640]

18. Rossmanith WG, Hoffmeister U, Wolfahrt S, et al. Expression and functional analysis of endothelial nitric oxide synthase (eNOS) in human placenta. Mol Hum Reprod. 1999; 5:487-494. [PubMed: 10338373]

19. Odibo AO, Nelson D, Stamilio DM, et al. Advanced maternal age is an independent risk factor for intrauterine growth restriction. Am J Perinat. 2006; 5:325-328.

20. Kirchengast S, Hartmann B. Advanced maternal age is not only associated with newborn somatometrics but also with the mode of delivery. Ann Hum Biol. 2003; 30:1-12. [PubMed: 12519651]

21. Mata-Greenwood E, Liao WX, Zheng J. Differential activation of multiple signaling pathways dictaes eNOS upregulation by FGF2 but no VEGF in placental artery endothelial cells. Placenta. 2008; 29:708-717. [PubMed: 18571718]

22. Lee CN, Chang SW, Cho NH, et al. Nitrous oxide synthase expression in placenta of preeclampsia. JKMS. 1997; 12:532-538.

\section{Appendix}

In addition to the authors, other members of the Eunice Kennedy Shriver National Institute of Child Health and Human Development Maternal-Fetal Medicine Units Network are as follows:

Wake Forest University Health Sciences - M. Harper, P. Meis, M. Swain, and C. Moorefield

Drexel University - M. DiVito, A. Sciscione, V. Berghella, M. Pollock, and M. Talucci 
Wayne State University - M. Dombrowski, G. Norman, A. Millinder, C. Sudz, and D. Driscoll

The Ohio State University - F. Johnson, J. Iams, M. Landon, S. Meadows, and P. Shubert

University of Utah - M. Varner, K. Anderson, A. Guzman, A. Crowley, and M. Fuller Northwestern University - G. Mallett

University of Texas Southwestern Medical Center - D. Weightman, L. Fay-Randall, and P. Mesa

University of Pittsburgh - T. Kamon, K. Lain, and M. Cotroneo

Columbia University - F. Malone, V. Pemberton, and S. Bousleiman

Case Western Reserve University - P. Catalano, C. Milluzzi, and C. Santori

University of North Carolina - K. Moise and K. Dorman

University of Chicago - A. Moawad, P. Jones and G. Mallett

University of Miami — D. Martin and F. Doyle

The University of Texas Health Science Center at Houston - L. Gilstrap and M.C. Day

Brown University - D. Allard and J. Tillinghast

University of Alabama at Birmingham - A. Northern, and K. Bailey

University of Cincinnati - H. How, N. Elder, B. Alexander, and W. Girdler

University of Tennessee - B. Mabie and R. Ramsey

The George Washington University Biostatistics Center - E. Thom, F. Galbis-Reig, and L. Leuchtenburg

Eunice Kennedy Shriver National Institute of Child Health and Human Development D. McNellis, K. Howell, and S. Tolivaisa 


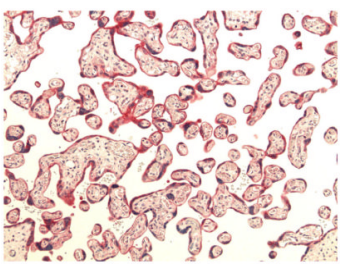

Figure 1.

Immunohistochemistry staining for eNOS in human placental tissue. 


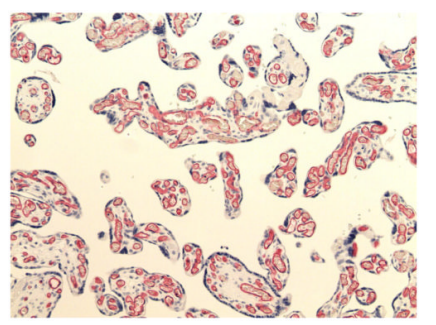

Figure 2.

Immunohistochemistry staining for CD34, an endothelial vascular stem cell antigen in human placental tissue. 


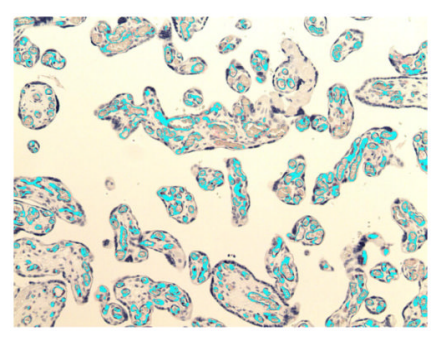

Figure 3.

Digital image quantification of CD34 immunostaining in human placental tissue. 
Table 1

Clinical characteristics of patients receiving single and multiple courses of antenatal betamethasone.

\begin{tabular}{|c|c|c|c|}
\hline Group & Single course $\mathbf{N}=91$ & Multiple courses $\mathbf{N}=100$ & p-value \\
\hline Maternal age (years) (mean $\pm \mathrm{SD})$ & $25.9(5.9)$ & $25.9(5.8)$ & 1.0 \\
\hline \multicolumn{4}{|l|}{ Ethnicity (number (\%)) } \\
\hline African American & $38(41.8)$ & $37(37.0)$ & 0.73 \\
\hline Caucasian & $29(31.9)$ & $37(37.0)$ & \\
\hline Hispanic/other & $24(26.4)$ & $26(26.0)$ & \\
\hline Maternal body mass index (mean \pm SD) & $26.4(7.0)$ & $26.1(6.3)$ & 0.94 \\
\hline Gestational age at randomization (weeks) (mean \pm SD) & $28.2(2.4)$ & $27.9(2.4)$ & 0.40 \\
\hline Gestational age at delivery (weeks) (mean \pm SD) & $35.2(4.1)$ & $35.0(3.8)$ & 0.71 \\
\hline Tobacco use (number [\%]) & $23(25.3)$ & $27(27.0)$ & 0.79 \\
\hline Nulliparous (number $[\%]$ ) & $8(8.8)$ & $10(10.0)$ & 0.78 \\
\hline Preterm labor/treatment with tocolytics (number [\%]) & $53(58.2)$ & $53(53.0)$ & 0.47 \\
\hline Maternal infection(number [\%]) & $31(34.1)$ & $26(26.0)$ & 0.22 \\
\hline Preterm premature rupture of membranes (number $[\%]$ ) & $14(15.4)$ & $15(15.0)$ & 0.94 \\
\hline Chorioamnionitis (number [\%]) & $3(3.3)$ & $3(3.0)$ & 1.0000 \\
\hline Abruption (number [\%]) & $8(8.9)$ & $8(8.8)$ & 0.83 \\
\hline
\end{tabular}


Table 2

Capillary area density and quantified eNOS expression in placentas of patients exposed to single and multiple courses of antenatal steroids

\begin{tabular}{|l|l|l|l|}
\hline Group & Single course N=91 & Multiple courses N=100 & p-value \\
\hline Maximum capillary density $($ mean \pm SD) & $7475.86(2340.10)$ & $7441.20(2048.16)$ & 0.72 \\
\hline Maximum eNOS staining (mean \pm SD) & $13258.14(3247.17)$ & $13154.66(3487.22)$ & 0.84 \\
\hline Mean capillary density (mean \pm SD) & $5807.09(1722.63)$ & $5820.92(1655.43)$ & 0.73 \\
\hline Mean eNOS staining (mean \pm SD) & $11028.49(2528.29)$ & $10903.94(2658.54)$ & 0.76 \\
\hline Ratio of eNOS to capillary density (mean \pm SD) & $2.08(0.82)$ & $2.05(0.82)$ & 0.74 \\
\hline
\end{tabular}




\section{Table 3}

Capillary area density and quantified eNOS expression for patients exposed to four courses versus one to three study courses of betamethasone in the active group.

\begin{tabular}{|l|c|c|c|}
\hline Group & $\mathbf{1}$ to 3 Courses N=32 & $\mathbf{4}$ Courses N=68 & p-value \\
\hline Maximum capillary density $($ mean \pm SD) & $6715.4(1784.5)$ & $7782.7(2086.9)$ & 0.005 \\
\hline Maximum eNOS staining (mean \pm SD) & $13571.2(3361.7)$ & $12955.7(3553.1)$ & 0.27 \\
\hline Mean capillary density $($ mean \pm SD) & $5244.3(1487.4)$ & $6092.3(1671.0)$ & 0.013 \\
\hline Mean eNOS staining (mean \pm SD) & $11293.4(2652.2)$ & $10717.9(2661.2)$ & 0.21 \\
\hline Ratio of eNOS to capillary density $($ mean \pm SD) & $2.33(0.84)$ & $1.92(0.78)$ & 0.02 \\
\hline
\end{tabular}

